\section{Reza Tabrizchi}

Division of Basic Medical Sciences, Faculty of Medicine, Memorial University of Newfoundland, St John's, NL, Canada

\section{Risk of cardiovascular events and cyclooxygenase-2 inhibitors}

Selective cyclooxygenase-2 (Cox-2) inhibitors have been at the centre of public attention since the revelation that the use of these drugs is associated with an increased risk of cardiovascular events (Tabrizchi 2005). Certainly pharmaco vigilance has an important place in the public domain and any new therapeutic information that leads to an increased level of physician-patients awareness has to be received with high degree of priority.

Recently, Caldwell and colleagues (2006) provided a systemic review and metaanalysis of double-blind, randomized, controlled studies of celecoxib to assess the risk of cardiovascular events. The primary goal was a comparative assessment of celecoxib with placebo, whereas the secondary analysis evaluated celecoxib versus placebo together with other non-steroidal antiinflammatory drugs (eg, diclofenac, ibuprofen, and paracetamol). Six studies were included in this analysis that met the criteria with the total sample size of 4422 and 12780 for the primary and secondary analysis, respectively. Data was included from trials evaluating treatment of medical conditions including rheumatoid arthritis, osteoarthritis, Alzheimer's disease, and the prevention of colorectal adenoma. The authors conclude that the increased risk of myocardial infarction observed with the use of celecoxib is consistent with this class of drugs (Caldwell et al 2006).

While great emphasis has been placed on the incidence of cardiovascular events, the risk of vascular events is not evenly distributed between myocardial infarction and cerebrovascular events. The incidence for cardiovascular events in this is recent meta-analysis was revealed to be $1.13 \%(\mathrm{n}=2574)$ in subjects taking celecoxib in comparison with $0.41 \%(\mathrm{n}=1447)$ in subjects on placebo with an odds ratio (OR) of 2.26 (95\% confidence interval [CI] 1.0-5.1) for myocardial infarction. In contrast, the incidence of cerebrovascular events recorded was $0.86 \%(n=2775)$ in subjects on celecoxib versus $0.79 \%(\mathrm{n}=1647)$ in subjects taking placebo with an OR $1.0(95 \%$ CI 0.51-1.84) (Caldwell et al 2006). Thus the risk of crebrovascular events is not comparable with the risk of myocardial infarction with use of this drug.

This finding is not unique to celecoxib and has been reported for rofecoxib as well, where the combined relative risk of myocardial infarction for rofecoxib in comparison with control was 2.24 (95\% CI 1.24-4.02) (Jüni et al 2004). This contrasts with the occurrence of stroke in which the relative risk was 1.02 (95\% CI 0.54-1.93) for rofecoxib versus control (Jüni et al 2004).

This class of drugs produce their beneficial effects by selective inhibition of the Cox enzyme. Cox-1 and Cox-2 are the isozymes involved in the catabolism of eicosanoids from arachidonic acid. Within the cascade responsible for the production of the various eicosanoids are two metabolites, prostaglandin $\mathrm{I}_{2}\left(\mathrm{PGI}_{2}\right)$ and thromboxane $\mathrm{A}_{2}\left(\mathrm{TxA}_{2}\right)$ which have critical roles on blood vessel function. While the latter compound is a potent vasoconstrictor and is a promoter of platelet aggregation, the former is a vasodilator and an inhibitor of platelet aggregation (Catella-Lawson 2001). However, it is also believed that Cox-2 is the enzyme mainly responsible for the production of $\mathrm{PGI}_{2}$ while Cox-1 plays a more central role in the biosynthesis of $\mathrm{TxA}_{2}$ (Vinals et al 1997; Brock et al 1999; Catella-Lawson et al 1999; McAdam et al 1999). Therefore, it is prudent to assume that selective inhibition of Cox-2 could change the balance between the production of $\mathrm{PGI}_{2}$ and $\mathrm{TxA}_{2}$ shifting 
it towards a higher levels of the latter molecule. This would be expected to increase the risk of vasospasm and thromboembolism and thus increase the risk of cardiovascular events (ie, myocardial infarction).

Surprisingly, it seems that while there is some evidence to suggest that the use of Cox-2 inhibitors such as rofecoxib and celecoxib increase the incidence of myocardial infarction, the same cannot be said about cerebrovascular events (Jüni et al 2004; Caldwell et al 2006). There are two important questions that readily come to mind. First, is the observation that therapy with selective Cox-2 inhibitors increases the risk of myocardial infarction, but not that of stroke real? Second, if the latter concept is true, what is the basis of this difference? Certainly, there are functional differences between brain and heart blood vessels. It is also recognized that coronary blood vessels experience excessive wall stress and it is possible that overall they are more sensitive to the reduction in the levels of $\mathrm{PGI}_{2}$ as well as being more sensitive to the detrimental actions of $\mathrm{TxA}_{2}$ during the inhibition of Cox-2 enzyme. Alternatively, cerebral blood vessels may have the inherent ability to overcome the selective inhibition exhibited by Cox-2 inhibitors and thus have the capability to restore the functional imbalance that occurs following the reduction in $\mathrm{PGI}_{2}$ and provide the counter balance to overcome the physiological effects of $\mathrm{TxA}_{2}$. Moreover, a combination of different structural and metabolic demands placed on each vessel together with differences in the functional behavior as well as the location of each vessel forms the basis for this disparity and in making one vessel less at risk of vascular events in contrast to the other. Clearly there is inadequate information for a succinct answer to this anomaly, and obviously the issue requires further in-depth investigation.

\section{References}

Brock TG, McNish RW, Peters-Golden M. 1999. Arachidonic acid is preferentially metabolized by cyclooxygenase- 2 to prostacyclin and prostaglandin $\mathrm{E}_{2} . J$ Biol Chem, 274:11660-6.

Caldwell B, Aldington S, Weatherall M, et al. 2006. Risk of cardiovascular events and celecoxib: a systematic review and meta-analysis. $J R S O c$ Med, 99:132-40.

Catella-Lawson F, McAdam B, Morrison BW, et al. 1999. Effects of specific inhibition of cyclooxygenase-2 on sodium balance, hemodynamics, and vasoactive eicosanoids. $J$ Pharmacol Exp Ther, 289:735-41

Catella-Lawson F. 2001. Vascular biology of thrombosis. Neurology, 57(Suppl 2):S5-S7.

Jüni P, Nartey L, Reichenbach S, et al. 2004. Risk of cardiovascular events and rofecoxib: cumulative meta-analysis. Lancet, 364:2021-9.

McAdam BF, Catella-Lawson F, Mardini IA, et al. 1999. Systemic biosynthesis of prostacyclin by cyclooxygenase (COX)-2: the human pharmacology of a selective inhibitor of COX-2. Proc Natl Acad Sci U S A, 96:272-7.

Tabrizchi R. 2005. Coxibs: can this class of drugs survive? Vasc Health Risk Manag, 1:5-8.

Vinals M, Martinez-Gonzalez J, Badimon JJ, et al. 1997. HDL-induced prostacyclin release in smooth muscle cells is dependent on cyclooxygenase-2 (Cox-2). Arterioscler Thromb Vasc Biol, 17:3481-8. 